Bull. Korean Math. Soc. 51 (2014), No. 1, pp. 267-285

http://dx.doi.org/10.4134/BKMS.2014.51.1.267

\title{
AN UPSTREAM PSEUDOSTRESS-VELOCITY MIXED FORMULATION FOR THE OSEEN EQUATIONS
}

\author{
Eun-Jae Park and Boyoon Seo
}

\begin{abstract}
An upstream scheme based on the pseudostress-velocity mixed formulation is studied to solve convection-dominated Oseen equations. Lagrange multipliers are introduced to treat the trace-free constraint and the lowest order Raviart-Thomas finite element space on rectangular mesh is used. Error analysis for several quantities of interest is given. Particularly, first-order convergence in $L^{2}$ norm for the velocity is proved. Finally, numerical experiments for various cases are presented to show the efficiency of this method.
\end{abstract}

\section{Introduction}

We consider the following Oseen equations

$$
\left\{\begin{array}{l}
\alpha \mathbf{u}-\nu \triangle \mathbf{u}+\mathbf{b} \cdot \nabla \mathbf{u}+\nabla p=\mathbf{f} \text { in } \Omega \\
\operatorname{div} \mathbf{u}=0 \text { in } \Omega \\
\mathbf{u}=\mathbf{0} \text { on } \partial \Omega
\end{array}\right.
$$

where $\Omega$ is an axis parallel domain in $\mathbb{R}^{2}$ with Lipschitz continuous boundary $\partial \Omega$. Let $\mathbf{f}=\left(f_{1}, f_{2}\right)$ and $\nu>0$ be the given external body force and kinematic viscosity, respectively. Denote $\mathbf{u}$ and $p$ to be the velocity vector and pressure, respectively. For simplicity, we assume that $\alpha>0$ and $\operatorname{div} \mathbf{b}=0$ and $\mathbf{b} \in$ $W^{1, \infty}(\Omega)^{2}$. Here we use the standard Sobolev spaces.

The Oseen problem occurs as linearized Navier-Stokes equations or often arises from an iterative procedure such as Picard's iteration [15]. Generally, the Oseen equations are convection-dominated and standard centered difference schemes or piecewise linear approximations produce spurious numerical oscillations. In the case of convection problem, upwind or upstream weighting

Received March 20, 2013.

2010 Mathematics Subject Classification. Primary 58B34, 58J42, 81T75.

Key words and phrases. pseudostress-velocity formulation, upstream scheme, mixed finite element, Oseen equations.

This research was supported by Basic Science Research Program through the National Research Foundation of Korea (NRF) funded by the Ministry of Education, Science and Technology NRF-2012R1A2A2A01046471. 
technique can be used [18]. There are various ways to treat this problem (see, for example, $[2,5,6])$.

The mixed finite element method has been successfully applied to several areas of interest, in particular, fluid flows in porous media. This is mainly due to the fact that the mixed method satisfies local mass conservation property and provides accurate fluxes whose normal components are continuous across inter-element boundaries. Mixed methods for linear and nonlinear second order elliptic problems are studied in $[4,20,22,23]$. The standard mixed finite element method to convection-dominated diffusion problems gives solutions with spurious oscillations. Hence we need to exploit an upstream weighting scheme for the convection term in the context of the mixed finite element method. This idea was developed by Jaffre [16] and by Dawson [12] for the scalar convectiondiffusion problem. The a posteriori error analysis of the upstream weighing mixed scheme was analyzed in [17]. There, it is shown that the a posteriori error estimator is not only reliable and efficient, but also computationally robust for several test problems.

The pseudostress-velocity formulation allows to use the Raviart-Thomas mixed finite approximation of the Stokes problem $[8,9,10,14]$. In this paper, we propose and analyze the upstream schemes based on the trace-free pseudostress and velocity formulation of the Oseen problem. Lagrange multipliers are introduced to treat the trace-free constraint. We use some of the ideas presented in [19] to obtain error bounds for velocity and pseudostress variables on rectangular mesh.

The remainder of this article is organized as follows. The pseudostressvelocity formulation is derived in the next section. In Section 3, we introduce the upstream mixed element method for the Oseen problem. The convergence analysis is given in Section 4. Finally, numerical experiments are presented in the last section.

\section{Pseudostress-velocity formulation}

Let us describe some notations and then derive weak formulation. Denote $\mathbb{M}_{2}$ to be the field of $2 \times 2$ matrix functions.

For vector functions $\mathbf{v}=\left(v_{1}, v_{2}\right)^{T}$ and $\mathbf{b}=\left(b_{1}, b_{2}\right)^{T}$, define its gradient $\nabla \mathbf{v} \in \mathbb{M}_{2}$ as a tensor and $\mathbf{b} \cdot \nabla \mathbf{v}$ as a vector:

$$
\nabla \mathbf{v}=\left(\begin{array}{cc}
\frac{\partial v_{1}}{\partial x} & \frac{\partial v_{1}}{\partial y} \\
\frac{\partial v_{2}}{\partial x} & \frac{\partial v_{2}}{\partial y}
\end{array}\right), \quad \mathbf{b} \cdot \nabla \mathbf{v}=\left(\begin{array}{c}
b_{1} \frac{\partial v_{1}}{\partial x}+b_{2} \frac{\partial v_{1}}{\partial y} \\
b_{1} \frac{\partial v_{2}}{\partial x}+b_{2} \frac{\partial v_{2}}{\partial y}
\end{array}\right)
$$

For a tensor function $\boldsymbol{\tau}=\left(\tau_{i j}\right)_{2 \times 2}$, let $\boldsymbol{\tau}_{i}=\left(\tau_{i 1}, \tau_{i 2}\right)$ denote its $i$ th-row for $i=1,2$ and define its divergence, normal, and trace by

$$
\operatorname{div} \boldsymbol{\tau}=\left(\begin{array}{c}
\operatorname{div} \boldsymbol{\tau}_{1} \\
\operatorname{div} \boldsymbol{\tau}_{2}
\end{array}\right), \quad \mathbf{n} \cdot \boldsymbol{\tau}=\boldsymbol{\tau} \mathbf{n}=\left(\begin{array}{c}
\mathbf{n} \cdot \boldsymbol{\tau}_{1} \\
\mathbf{n} \cdot \boldsymbol{\tau}_{2}
\end{array}\right), \quad \operatorname{tr} \boldsymbol{\tau}=\tau_{11}+\tau_{22},
$$


respectively. Let $\mathcal{A}: \mathbb{M}_{2} \rightarrow \mathbb{M}_{2}$ be a linear map defined by $\mathcal{A} \boldsymbol{\tau}=\boldsymbol{\tau}-\frac{1}{2}(\operatorname{tr} \boldsymbol{\tau}) \mathbf{I}$, where $\mathbf{I}$ is $2 \times 2$ identity matrix. Introducing the pseudostress variable

$$
\boldsymbol{\sigma}=\nu \nabla \mathbf{u}-p \mathbf{I}
$$

system (1) can be written as

$$
\left\{\begin{array}{l}
\kappa \mathcal{A} \boldsymbol{\sigma}-\nabla \mathbf{u}=0 \\
\operatorname{div} \boldsymbol{\sigma}-\mathbf{b} \cdot \nabla \mathbf{u}-\alpha \mathbf{u}=-\mathbf{f}
\end{array}\right.
$$

where $\kappa=1 / \nu$. Indeed, notice that $\mathcal{A} \boldsymbol{\sigma}$ is trace free, hence the incompressibility constraint $\operatorname{div} \mathbf{u}=0$ is satisfied through $\operatorname{div} \mathbf{u}=\operatorname{tr}(\nabla \mathbf{u})=0$. Also, the pressure

$$
p=-\frac{1}{2} \operatorname{tr} \sigma
$$

is unique up to a constant. It is clear that the Oseen equations have a unique solution provided that $\int_{\Omega} p=0$, which implies

$$
\int_{\Omega} \operatorname{tr} \sigma=0
$$

So, we use the following function spaces:

$$
\begin{aligned}
& \mathbf{H}:=\mathbf{H}(\operatorname{div} ; \Omega)=H(\operatorname{div} ; \Omega)^{2}, \\
& \boldsymbol{\Sigma}:=\left\{\boldsymbol{\tau} \in \mathbf{H} \mid \int_{\Omega} \operatorname{tr} \boldsymbol{\tau}=0\right\}, \\
& \mathbf{V}:=\mathbf{L}^{2}(\Omega)=L^{2}(\Omega)^{2},
\end{aligned}
$$

where $H(\operatorname{div} ; \Omega)=\left\{\mathbf{v} \in L^{2}(\Omega)^{2} \mid \operatorname{div} \mathbf{v} \in L^{2}(\Omega)\right\}$. The simple variational problem of the pseudostress-velocity formulation is to find a pair $(\boldsymbol{\sigma}, \mathbf{u}) \in \mathbf{\Sigma} \times \mathbf{V}$ such that

$$
\begin{cases}(\kappa \mathcal{A} \boldsymbol{\sigma}, \boldsymbol{\tau})+(\operatorname{div} \boldsymbol{\tau}, \mathbf{u})=0, & \forall \boldsymbol{\tau} \in \mathbf{\Sigma} \\ (\operatorname{div} \boldsymbol{\sigma}, \mathbf{v})-G(\mathbf{u}, \mathbf{v})=-(\mathbf{f}, \mathbf{v}), & \forall \mathbf{v} \in \mathbf{V}\end{cases}
$$

where $G(\mathbf{u}, \mathbf{v})=(\mathbf{b} \cdot \nabla \mathbf{u}, \mathbf{v})+(\alpha \mathbf{u}, \mathbf{v})$. Here, the inner product $(\boldsymbol{\sigma}, \boldsymbol{\tau})$ for tensor functions is $\int_{\Omega} \boldsymbol{\sigma}: \boldsymbol{\tau}$ and $(\mathbf{u}, \mathbf{v})=\int_{\Omega} \mathbf{u} \cdot \mathbf{v}$ for vector functions. But, this weak formulation give us difficulties in error analysis and taking the basis for finite element space of $\boldsymbol{\Sigma}$. So, we introduce a Lagrange multiplier to satisfy the tracefree condition (4). The following weak form is equivalent to (5): Find a pair $(\boldsymbol{\sigma}, \mathbf{u}, \ell) \in \mathbf{H} \times \mathbf{V} \times \mathbb{R}$ such that

$$
\begin{cases}(\kappa \mathcal{A} \boldsymbol{\sigma}, \boldsymbol{\tau})+(\operatorname{div} \boldsymbol{\tau}, \mathbf{u})+d(\boldsymbol{\tau}, \ell)=0, & \forall \boldsymbol{\tau} \in \mathbf{H}, \\ (\operatorname{div} \boldsymbol{\sigma}, \mathbf{v})-G(\mathbf{u}, \mathbf{v})=-(\mathbf{f}, \mathbf{v}), & \forall \mathbf{v} \in \mathbf{V}, \\ d(\boldsymbol{\sigma}, \mu)=0, & \forall \mu \in \mathbb{R},\end{cases}
$$

where $d(\tau, \ell)=\ell \int_{\Omega} \operatorname{tr} \tau$. We will use the following lemma whose proof can be found in $[4,7]$. 
Lemma 2.1. For any $\tau \in \Sigma$, we have

$$
\|\operatorname{tr} \boldsymbol{\tau}\| \leq C\left(\|\mathcal{A} \boldsymbol{\tau}\|+\|\operatorname{div} \boldsymbol{\tau}\|_{-1}\right) .
$$

Note that

$$
\|\boldsymbol{\tau}\|^{2}=\|\mathcal{A} \tau\|^{2}+\frac{1}{2}\|\operatorname{tr} \boldsymbol{\tau}\|^{2}
$$

which, together with Lemma 2.1, implies

$$
\|\boldsymbol{\tau}\| \leq C\left(\|\mathcal{A} \tau\|+\|\operatorname{div} \tau\|_{-1}\right) \leq C(\|\mathcal{A} \tau\|+\|\operatorname{div} \tau\|) .
$$

\section{The upstream mixed element method}

Let $\mathcal{R}_{h}=\left\{R_{i, j}: 0 \leq i \leq n-1,0 \leq j \leq m-1\right\}$ be a quasi-uniform partition of the domain $\Omega=(a, b) \times(c, d)$ into a union of rectangle $R_{i, j}:=$ $\left[x_{i}, x_{i+1}\right] \times\left[y_{j}, y_{j+1}\right]$ based on axes partitions:

$$
\begin{aligned}
& a=x_{0}<x_{1}<\cdots<x_{n}=b, \\
& c=y_{0}<y_{1}<\cdots<y_{m}=d .
\end{aligned}
$$

Let $h_{i}^{x}=x_{i+1}-x_{i}, h_{j}^{y}=y_{j+1}-y_{j}$ and its area as $\left|R_{i, j}\right|$ and denote four edges as follows

$$
\begin{array}{cl}
e_{i}^{x}=\left\{\left(x_{i}, y\right): y_{j}<y<y_{j+1}\right\}, & e_{i+1}^{x}=\left\{\left(x_{i+1}, y\right): y_{j}<y<y_{j+1}\right\}, \\
e_{j}^{y}=\left\{\left(x, y_{j}\right): x_{i}<x<x_{i+1}\right\}, & e_{j+1}^{y}=\left\{\left(x, y_{j+1}\right): x_{i}<x<x_{i+1}\right\} .
\end{array}
$$

Let $h$ be the largest mesh size of the rectangluation, i.e., $h=\max _{i, j}\left\{h_{i}^{x}, h_{j}^{y}\right\}$. The partition $\mathcal{R}_{h}$ is quasi-uniform, which means that there exist two constants $C_{1}, C_{2}$ such that

$$
C_{1} h^{2} \leq\left|R_{i, j}\right| \leq C_{2} h^{2} .
$$

We define mixed finite element space $\mathbf{H}_{h} \times \mathbf{V}_{h} \subset \mathbf{H} \times \mathbf{V}$ based on the lowest Raviart-Thomas space. For $Q_{i}^{x}=R_{i-1, j} \cup R_{i, j}$ if $R_{i-1, j}$ or $R_{i, j}$ exists, define scalar function $\phi_{i}^{x}$ on $Q_{i}^{x}$ as follows

$$
\phi_{i}^{x}(x, y)=\left\{\begin{array}{cl}
\frac{x-x_{i-1}}{h_{i-1}^{x}} & \text { if }(x, y) \in R_{i-1, j}, \\
\frac{x_{i+1}-x}{h_{i}^{x}} & \text { if }(x, y) \in R_{i, j}, \\
0 & \text { otherwise. }
\end{array}\right.
$$

Similarly, for $Q_{j}^{y}=R_{i, j-1} \cup R_{i, j}$ if $R_{i, j-1}$ or $R_{i, j}$ exists, define function $\phi_{j}^{y}$ on $Q_{j}^{y}$ as follows

$$
\phi_{j}^{y}(x, y)=\left\{\begin{array}{cl}
\frac{y-y_{j-1}}{h_{j-1}^{y}} & \text { if }(x, y) \in R_{i, j-1}, \\
\frac{y_{j+1}-y}{h_{j}^{y}} & \text { if }(x, y) \in R_{i, j}, \\
0 & \text { otherwise. }
\end{array}\right.
$$


Then, we define finite element spaces as

$$
\begin{aligned}
\mathbf{H}_{h}: & =\mathbf{R T}_{0}=\left\{\boldsymbol{\tau} \in \mathbf{H}(\mathbf{d i v} ; \Omega):\left.\boldsymbol{\tau}\right|_{R} \in \mathbf{R T}_{0}(R), \forall R \in \mathcal{R}_{h}\right\} \\
& =\operatorname{span}\left\{\left(\begin{array}{cc}
\phi_{i}^{x} & 0 \\
0 & 0
\end{array}\right),\left(\begin{array}{cc}
0 & \phi_{j}^{y} \\
0 & 0
\end{array}\right),\left(\begin{array}{cc}
0 & 0 \\
\phi_{i}^{x} & 0
\end{array}\right),\left(\begin{array}{cc}
0 & 0 \\
0 & \phi_{j}^{y}
\end{array}\right)\right\}
\end{aligned}
$$

and

$$
\mathbf{V}_{h}:=\operatorname{span}\left\{\left(\begin{array}{l}
1 \\
0
\end{array}\right),\left(\begin{array}{l}
0 \\
1
\end{array}\right)\right\}
$$

Note that each row of tensor function in $\mathbf{H}_{h}$ satisfies the continuity of the normal component of vector field at interfaces of elements.

For exposition of upstream schemes some notations are in order. We will represent normal vector as two ways. First, for given edge $e$ of an element $R \in \mathcal{R}_{h}$ we assign a unit normal vector $\mathbf{n}_{e}$, which is the same as the $x$-direction or $y$-direction. Then, given a pair $\left(e, \mathbf{n}_{e}\right)$ with an interior edge, one can uniquely define the neighboring elements $R_{e}^{+}$and $R_{e}^{-}$with common edge $e$ so that $\mathbf{n}_{e}$ points toward $R_{e}^{+}$. Second, for given element $R$ a vector $\mathbf{n}$ will be considered "outward" to an underlying element as in Figure 1.

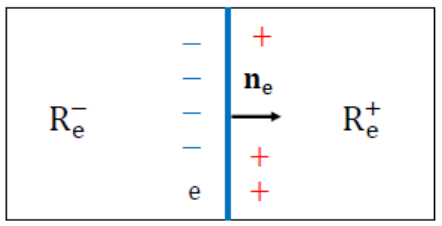

b

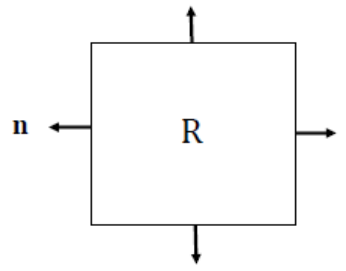

(b)

Figure 1. (a) edge-based normal vector and elements (b) element-based outward unit normal vector

We define $(\mathbf{b} \cdot \mathbf{n})^{+}=\max \{\mathbf{b} \cdot \mathbf{n}, 0\},(\mathbf{b} \cdot \mathbf{n})^{-}=\min \{\mathbf{b} \cdot \mathbf{n}, 0\}$. For given element $R,\left(\mathbf{u}^{h}\right)^{\text {int }}$ stands for the trace of $\mathbf{u}^{h}$ on $\partial R$ from the interior of $R$ and $\left(\mathbf{u}^{h}\right)^{\text {ext }}$ is that from the exterior of $R$. We set the exterior trace on $\partial R \cap \partial \Omega$ to be 0 .

Now, we define an upstream mixed finite element approximation of $G(\mathbf{u}, \mathbf{v})$ in (6) as follows:

$$
G_{h}(\mathbf{u}, \mathbf{v})=\sum_{R \in \mathcal{R}_{h}} \int_{\partial R}\left((\mathbf{b} \cdot \mathbf{n})^{+}\left(\mathbf{u}^{h}\right)^{\mathrm{int}}+(\mathbf{b} \cdot \mathbf{n})^{-}\left(\mathbf{u}^{h}\right)^{\mathrm{ext}}\right) \cdot \mathbf{v}^{h} d s+(\alpha \mathbf{u}, \mathbf{v})
$$


Our upstream mixed finite element approximation of the weak formulation (6) is to find a pair $\left(\boldsymbol{\sigma}^{h}, \mathbf{u}^{h}, \ell^{h}\right) \in \mathbf{H}_{h} \times \mathbf{V}_{h} \times \mathbb{R}$ such that

$$
\begin{cases}\left(\kappa \mathcal{A} \boldsymbol{\sigma}^{h}, \boldsymbol{\tau}^{h}\right)+\left(\boldsymbol{d i v} \boldsymbol{\tau}^{h}, \mathbf{u}^{h}\right)+d\left(\boldsymbol{\tau}^{h}, \ell^{h}\right)=0, & \forall \boldsymbol{\tau}^{h} \in \mathbf{H}_{h}, \\ \left(\boldsymbol{d i v} \boldsymbol{\sigma}^{h}, \mathbf{v}^{h}\right)-G_{h}\left(\mathbf{u}^{h}, \mathbf{v}^{h}\right)=-\left(\mathbf{f}, \mathbf{v}^{h}\right), & \forall \mathbf{v}^{h} \in \mathbf{V}_{h}, \\ d\left(\boldsymbol{\sigma}^{h}, \mu^{h}\right)=0, & \forall \mu^{h} \in \mathbb{R} .\end{cases}
$$

Lemma 3.1. Let $\varepsilon$ be the collection of interior edges. Then the bilinear form $G_{h}(\mathbf{u}, \mathbf{v})$ can be rewritten as follows:

$$
\begin{aligned}
G_{h}(\mathbf{u}, \mathbf{v})= & \frac{1}{2} \sum_{e \in \varepsilon} \int_{e}|\mathbf{b} \cdot \mathbf{n}|([\mathbf{u}] \cdot[\mathbf{v}]) d s+(\alpha \mathbf{u}, \mathbf{v}) \\
& +\frac{1}{2} \sum_{e \in \varepsilon} \int_{e}\left(\mathbf{b} \cdot \mathbf{n}_{\mathbf{e}}\right)\left(\mathbf{u}_{R_{e}^{+}}+\mathbf{u}_{R_{e}^{-}}\right) \cdot\left(\mathbf{v}_{R_{e}^{-}}-\mathbf{v}_{R_{e}^{+}}\right) d s, \quad \forall \mathbf{v} \in \mathbf{V}_{h},
\end{aligned}
$$

where $[\mathbf{u}]$ denotes the jump of $\mathbf{u}$ on edge $e$.

Proof. The idea is to rewrite the corresponding sums over the edges. For given interior edge $e$ and a pre-assigned unit normal vector $\mathbf{n}_{e}$, there are two elements $R_{e}^{+}$and $R_{e}^{-}$. We assume that $R_{e}^{+}$contribute with the vector $\mathbf{n} \equiv-\mathbf{n}_{e}$ and $R_{e}^{-}$ contribute with the vector $\mathbf{n} \equiv \mathbf{n}_{e}$ as reference to Figure 1. See [11] for a complete proof.

Lemma 3.2. For any $\mathbf{v} \in \mathbf{V}_{h}$, the bilinear form $G_{h}(\mathbf{v}, \mathbf{v})$ satisfies:

$$
G_{h}(\mathbf{v}, \mathbf{v})=(\alpha \mathbf{v}, \mathbf{v})+\frac{1}{2}\|\mathbf{v}\|^{2}
$$

where $\|\mathbf{v}\|^{2}=\sum_{e \in \varepsilon} \int_{e}|\mathbf{b} \cdot \mathbf{n}|([\mathbf{v}] \cdot[\mathbf{v}]) d s$.

Proof. It follows from Lemma 3.1 that

$$
\begin{aligned}
& \frac{1}{2} \sum_{e \in \varepsilon} \int_{e}\left(\mathbf{b} \cdot \mathbf{n}_{e}\right)\left(\mathbf{v}_{R_{e}^{+}}+\mathbf{v}_{R_{e}^{-}}\right) \cdot\left(\mathbf{v}_{R_{e}^{-}}-\mathbf{v}_{R_{e}^{+}}\right) d s \\
= & \frac{1}{2} \sum_{e \in \varepsilon} \int_{e}\left(\mathbf{b} \cdot \mathbf{n}_{e}\right)\left[\left|\mathbf{v}_{R_{e}^{-}}\right|^{2}-\left|\mathbf{v}_{R_{e}^{+}}\right|^{2}\right] d s \\
= & \frac{1}{2} \sum_{R \in \mathcal{R}_{h}} \int_{\partial R}(\mathbf{b} \cdot \mathbf{n}) \mathbf{v} \cdot \mathbf{v} d s \\
= & \frac{1}{2} \sum_{R \in \mathcal{R}_{h}} \int_{R} \operatorname{div} \mathbf{b}(\mathbf{v} \cdot \mathbf{v}) d x d y=0 .
\end{aligned}
$$

The last identity follows from the incompressibility condition divb $=0$.

Now we are ready to prove unique solvability of our discrete system.

Theorem 3.3. For sufficiently small $h$, there exists a unique solution $\left(\boldsymbol{\sigma}^{h}, \mathbf{u}^{h}\right.$, $\left.\ell^{h}\right)$ in $\mathbf{H}_{h} \times \mathbf{V}_{h} \times \mathbb{R}$ for system (8). 
Proof. It is sufficient to prove that the problem has just trivial solution when $\mathbf{f}=\mathbf{0}$. Selecting $\boldsymbol{\tau}^{h}=\boldsymbol{\sigma}^{h}, \mathbf{v}^{h}=\mathbf{u}^{h}$ and $\mu^{h}=\ell^{h}$ in (8), we get

$$
\left\{\begin{array}{l}
\left(\kappa \mathcal{A} \boldsymbol{\sigma}^{h}, \boldsymbol{\sigma}^{h}\right)+\left(\operatorname{div} \boldsymbol{\sigma}^{h}, \mathbf{u}^{h}\right)=0, \\
\left(\operatorname{div} \boldsymbol{\sigma}^{h}, \mathbf{u}^{h}\right)-G_{h}\left(\mathbf{u}^{h}, \mathbf{u}^{h}\right)=0 .
\end{array}\right.
$$

So, we have that

$$
\left(\kappa \mathcal{A} \boldsymbol{\sigma}^{h}, \boldsymbol{\sigma}^{h}\right)+G_{h}\left(\mathbf{u}^{h}, \mathbf{u}^{h}\right)=0 .
$$

Since $\left(\kappa \mathcal{A} \boldsymbol{\sigma}^{h}, \boldsymbol{\sigma}^{h}\right)=\kappa\left\|\mathcal{A} \boldsymbol{\sigma}^{h}\right\|^{2}$ and $G_{h}\left(\mathbf{u}^{h}, \mathbf{u}^{h}\right)=\alpha\left\|\mathbf{u}^{h}\right\|^{2}+\frac{1}{2}\left\|\mathbf{u}^{h}\right\|^{2}$, we have

$$
\left\|\mathcal{A} \boldsymbol{\sigma}^{h}\right\|=0 \text { and }\left\|\mathbf{u}^{h}\right\|=0 \text {. }
$$

Next, if we choose $\mathbf{v}^{h}=\operatorname{div} \boldsymbol{\sigma}^{h}$ in second equation of (8), we get from (7)

$$
\left\|\boldsymbol{\sigma}^{h}\right\|=0 \text {. }
$$

Finally, from the first equation in $(8)$

$$
d\left(\boldsymbol{\tau}^{h}, \ell^{h}\right)=-\left(\kappa \mathcal{A} \boldsymbol{\sigma}^{h}, \boldsymbol{\tau}^{h}\right)-\left(\operatorname{div} \boldsymbol{\tau}^{h}, \mathbf{u}^{h}\right)=0 .
$$

Choosing $\boldsymbol{\tau}^{h} \in \mathbf{H}_{h}$ with $\int_{\Omega} \operatorname{tr} \boldsymbol{\tau}^{h} \neq 0$, we have $\ell^{h}=0$ as required.

Remark 3.4. If we choose a constant tensor $\tau^{h}=\frac{1}{2|\Omega|}\left(\begin{array}{cc}\ell-\ell^{h} & 0 \\ 0 & \ell-\ell^{h}\end{array}\right)$, then $\tau^{h}$ belongs to $\mathbf{H}_{h}$ and satisfies that

$$
\ell-\ell^{h}=\int_{\Omega} \operatorname{tr} \tau^{h}
$$

Noting that $\mathcal{A} \tau^{h}=0$ and from error equation

$$
\begin{aligned}
\left(\ell-\ell^{h}\right)^{2} & =d\left(\boldsymbol{\tau}^{h}, \ell-\ell^{h}\right) \\
& =-\left(\kappa \mathcal{A}\left(\boldsymbol{\sigma}-\boldsymbol{\sigma}^{h}\right), \boldsymbol{\tau}^{h}\right)-\left(\operatorname{div} \boldsymbol{\tau}^{h}, \mathbf{u}-\mathbf{u}^{h}\right) \\
& =-\left(\kappa\left(\boldsymbol{\sigma}-\boldsymbol{\sigma}^{h}\right), \mathcal{A} \boldsymbol{\tau}^{h}\right)-\left(\operatorname{div} \boldsymbol{\tau}^{h}, \mathbf{u}-\mathbf{u}^{h}\right)=0,
\end{aligned}
$$

we must have $\ell=\ell_{h}$.

\section{Error analysis}

To estimate errors, we define a projection using mean value of integration. For a given $R_{i, j}$ and scalar function $p(x, y)$, put

$$
\bar{p}\left(x_{i}\right)=\frac{1}{h_{j}^{y}} \int_{y_{j}}^{y_{j+1}} p\left(x_{i}, y\right) d y
$$

and define an interpolation $\pi_{x} p$ for $(x, y) \in R_{i, j}$

$$
\pi_{x} p(x, y)=\bar{p}\left(x_{i}\right) \phi_{i}^{x}(x, y)+\bar{p}\left(x_{i+1}\right) \phi_{i+1}^{x}(x, y),
$$

which is piecewise constant in $y$ and piecewise linear in $x$. Similarly we define an interpolation $\pi_{y} p(x, y)$, which is piecewise constant in $x$ and piecewise linear in $y$. Define

$$
\Pi_{h}: H(\operatorname{div} ; R) \longrightarrow R T_{0}(R)
$$


by

$$
\Pi_{h} \mathbf{p}=\left(\pi_{x} p_{1}, \pi_{y} p_{2}\right) \quad \text { for } \mathbf{p}=\left(p_{1}, p_{2}\right) \in H(\operatorname{div} ; R) .
$$

Then, the projection satisfies the followings

$$
\int_{e}\left(\mathbf{p}-\Pi_{h} \mathbf{p}\right) \cdot \mathbf{n} d s=0 \quad \text { for each edge } e \in \partial R .
$$

Thus, we extend this projection to tensor $\boldsymbol{\sigma}=\left(\begin{array}{ll}\sigma_{11} & \sigma_{12} \\ \sigma_{21} & \sigma_{22}\end{array}\right) \in \mathbf{H}$, we define $\boldsymbol{\Pi}_{h} \boldsymbol{\sigma}$ as follows

$$
\left.\boldsymbol{\Pi}_{h} \boldsymbol{\sigma}\right|_{R}=\left(\begin{array}{cc}
\pi_{x} \sigma_{11} & \pi_{y} \sigma_{12} \\
\pi_{x} \sigma_{21} & \pi_{y} \sigma_{22}
\end{array}\right)
$$

Then,

$$
\Pi_{h} \boldsymbol{\sigma}:=\left.\sum_{R \in \mathcal{R}_{h}} \Pi_{h} \boldsymbol{\sigma}\right|_{R} .
$$

Next, we define a piecewise constant interpolation $P_{h} v$ for $v \in L^{2}(R)$ as follows

$$
P_{h} v=\frac{1}{|R|} \int_{R} v(x, y) d x d y .
$$

So, for $\mathbf{u}=\left(\begin{array}{l}u_{1} \\ u_{2}\end{array}\right) \in \mathbf{L}^{2}(\Omega)$, define an interpolation $\mathbf{P}_{h} \mathbf{u} \in \mathbf{V}_{h}$ as follows

$$
\mathbf{P}_{h} \mathbf{u}=\sum_{R \in \mathcal{R}_{h}}\left(\begin{array}{c}
P_{h} u_{1} \\
P_{h} u_{2}
\end{array}\right) .
$$

From (9), it is easy to check the validity of the commutativity property

$$
\operatorname{div} \Pi_{h} \boldsymbol{\tau}=\mathbf{P}_{h} \operatorname{div} \boldsymbol{\tau}, \quad \forall \boldsymbol{\tau} \in \mathbf{H} .
$$

Thus, we obtain convergence results for projections:

Lemma 4.1. For $\boldsymbol{\sigma} \in \mathbf{H}$, we have the following approximation properties

$$
\begin{aligned}
\left\|\boldsymbol{\sigma}-\boldsymbol{\Pi}_{h} \boldsymbol{\sigma}\right\| & \leq C h\|\boldsymbol{\sigma}\|_{1}, \\
\left\|\operatorname{div}\left(\boldsymbol{\sigma}-\boldsymbol{\Pi}_{h} \boldsymbol{\sigma}\right)\right\| & \leq C h\|\operatorname{div} \boldsymbol{\sigma}\|_{1} .
\end{aligned}
$$

Lemma 4.2. For $\boldsymbol{\sigma} \in \mathbf{H}$, the interpolation $\boldsymbol{\Pi}_{h} \boldsymbol{\sigma}$ satisfies the followings

$$
\left(\kappa \mathcal{A}\left(\boldsymbol{\sigma}-\boldsymbol{\Pi}_{h} \boldsymbol{\sigma}\right), \boldsymbol{\tau}\right) \leq \kappa C h\|\mathcal{A} \boldsymbol{\sigma}\|_{1}\|\boldsymbol{\tau}\|, \quad \forall \boldsymbol{\tau} \in \mathbf{H}_{h}
$$

and

$$
\left(\operatorname{div}\left(\boldsymbol{\sigma}-\boldsymbol{\Pi}_{h} \boldsymbol{\sigma}\right), \mathbf{v}\right)=0, \quad \forall \mathbf{v} \in \mathbf{V}_{h} .
$$

Proof. It follows from Lemma 4.1 and the commuting property $\mathcal{A} \Pi_{h} \boldsymbol{\sigma}=$ $\Pi_{h} \mathcal{A} \sigma$ that

$$
\left\|\mathcal{A}\left(\boldsymbol{\sigma}-\boldsymbol{\Pi}_{h} \boldsymbol{\sigma}\right)\right\| \leq\left\|\mathcal{A} \boldsymbol{\sigma}-\Pi_{h} \mathcal{A} \boldsymbol{\sigma}\right\| \leq C h\|\mathcal{A} \boldsymbol{\sigma}\|_{1} .
$$

Since $\mathbf{v} \in \mathbf{V}_{h}$ is constant on $R$, it follows from the definition of $\boldsymbol{\Pi}_{h} \boldsymbol{\sigma}$ that we have

$$
\int_{R} \mathbf{v} \cdot\left(\operatorname{div} \boldsymbol{\Pi}_{h} \boldsymbol{\sigma}\right) d x d y=\int_{\partial R} \mathbf{v} \cdot\left(\mathbf{n} \cdot \boldsymbol{\Pi}_{h} \boldsymbol{\sigma}\right) d s
$$




$$
=\int_{\partial R} \mathbf{v} \cdot(\mathbf{n} \cdot \boldsymbol{\sigma}) d s=\int_{R} \mathbf{v} \cdot(\operatorname{div} \boldsymbol{\sigma}) d x d y .
$$

Now, for the error analysis, define

$$
\boldsymbol{\xi}_{\sigma}=\Pi_{h} \boldsymbol{\sigma}-\boldsymbol{\sigma}^{h}, \quad \boldsymbol{\xi}_{\mathbf{u}}=\mathbf{P}_{h} \mathbf{u}-\mathbf{u}^{h}, \quad \eta_{\boldsymbol{\sigma}}=\boldsymbol{\sigma}-\Pi_{h} \boldsymbol{\sigma}, \quad \boldsymbol{\eta}_{\mathbf{u}}=\mathbf{u}-\mathbf{P}_{h} \mathbf{u} .
$$

Lemma 4.3. When $h$ is sufficiently small there is a positive constant $C$ independent of $h$ such that

$$
\begin{gathered}
{\left[\sum_{e_{i}^{x}}\left|\boldsymbol{\xi}_{\mathbf{u}, i, j}-\boldsymbol{\xi}_{\mathbf{u}, i-1, j}\right|^{2}\right]^{\frac{1}{2}} \leq \kappa C\left\|\mathcal{A} \boldsymbol{\xi}_{\boldsymbol{\sigma}}\right\|+\kappa C h\|\boldsymbol{\sigma}\|_{1},} \\
{\left[\sum_{e_{j}^{y}}\left|\boldsymbol{\xi}_{\mathbf{u}, i, j}-\boldsymbol{\xi}_{\mathbf{u}, i, j-1}\right|^{2}\right]^{\frac{1}{2}} \leq \kappa C\left\|\mathcal{A} \boldsymbol{\xi}_{\boldsymbol{\sigma}}\right\|+\kappa C h\|\boldsymbol{\sigma}\|_{1},}
\end{gathered}
$$

where $\boldsymbol{\xi}_{\mathbf{u}}=\left(\begin{array}{l}\xi_{u_{1}} \\ \xi_{u_{2}}\end{array}\right)$ and $\boldsymbol{\xi}_{\boldsymbol{\sigma}}=\left(\begin{array}{ll}\xi_{\sigma_{11}} & \xi_{\sigma_{12}} \\ \xi_{\sigma_{21}} & \xi_{\sigma_{22}}\end{array}\right)$.

Proof. Let $Q_{i}^{x}=R_{i-1, j} \cup R_{i, j}$. Selecting $\tau=\left(\begin{array}{cc}\phi_{i}^{x} & 0 \\ 0 & 0\end{array}\right)$ in (6) and (8) we have that

$$
\begin{aligned}
\kappa \int_{Q_{i}^{x}}\left(\sigma_{11}-\frac{1}{2} \operatorname{tr} \sigma\right) \phi_{i}^{x} & =\int_{Q_{i}^{x}} \frac{\partial \phi_{i}^{x}}{\partial x} u_{1}-\ell \int_{Q_{i}^{x}} \phi_{i}^{x} \\
& =h_{j}^{y}\left(P_{h} u_{1, i-1, j}-P_{h} u_{1, i, j}\right)-\ell \int_{Q_{i}^{x}} \phi_{i}^{x}
\end{aligned}
$$

and

$$
\begin{aligned}
\kappa \int_{Q_{i}^{x}}\left(\sigma_{11}^{h}-\frac{1}{2} \operatorname{tr} \sigma^{h}\right) \phi_{i}^{x} & =\int_{Q_{i}^{x}} \frac{\partial \phi_{i}^{x}}{\partial x} u_{1}^{h}-\ell^{h} \int_{Q_{i}^{x}} \phi_{i}^{x} \\
& =h_{j}^{y}\left(u_{1, i-1, j}^{h}-u_{1, i, j}^{h}\right)-\ell^{h} \int_{Q_{i}^{x}} \phi_{i}^{x} .
\end{aligned}
$$

Note that

$$
\begin{aligned}
\kappa \int_{Q_{i}^{x}}\left(\left(\sigma_{11}-\sigma_{11}^{h}\right)-\frac{1}{2}\left(\operatorname{tr} \boldsymbol{\sigma}-\operatorname{tr} \boldsymbol{\sigma}^{h}\right)\right) \phi_{i}^{x} & \leq \kappa \int_{Q_{i}^{x}}\left|\mathcal{A} \sigma_{11}-\mathcal{A} \sigma_{11}^{h}\right| \\
& \leq \kappa \int_{Q_{i}^{x}}\left(\left|\mathcal{A} \xi_{\sigma_{11}}\right|+\left|\mathcal{A} \eta_{\sigma_{11}}\right|\right) .
\end{aligned}
$$

Since $\ell=\ell^{h}$, by subtracting (18) from (19), we have that

$$
\left|\xi_{u_{1}, i, j}-\xi_{u_{1}, i-1, j}\right| \leq \kappa \frac{1}{h_{j}^{y}} \int_{Q_{i}^{x}}\left|\mathcal{A} \xi_{\sigma_{11}}\right|+\kappa C \frac{1}{h_{j}^{y}} \int_{Q_{i}^{x}}\left|\mathcal{A} \eta_{\sigma_{11}}\right| .
$$


So, using $(a+b)^{2} \leq 2\left(a^{2}+b^{2}\right)$ and the Hölder inequality,

$$
\begin{aligned}
& \sum_{e_{i}^{x}}\left(\xi_{u_{1}, i, j}-\xi_{u_{1}, i-1, j}\right)^{2} \\
\leq & C \sum_{e_{i}^{x}}\left(\kappa^{2} \frac{\left|Q_{i}^{x}\right|}{\left(h_{j}^{y}\right)^{2}} \int_{Q_{i}^{x}}\left|\mathcal{A} \xi_{\sigma_{11}}\right|^{2}+\kappa^{2} \frac{\left|Q_{i}^{x}\right|}{\left(h_{j}^{y}\right)^{2}} \int_{Q_{i}^{x}}\left|\mathcal{A} \eta_{\sigma_{11}}\right|^{2}\right) \\
\leq & \kappa^{2} C\left\|\mathcal{A} \boldsymbol{\xi}_{\boldsymbol{\sigma}}\right\|^{2}+\kappa^{2} C h^{2}\|\boldsymbol{\sigma}\|_{1}^{2} .
\end{aligned}
$$

If we select $\boldsymbol{\tau}=\left(\begin{array}{cc}0 & 0 \\ \phi_{i}^{x} & 0\end{array}\right)$, we have that

$$
\sum_{e_{i}^{x}}\left(\xi_{u_{2}, i, j}-\xi_{u_{2}, i-1, j}\right)^{2} \leq \kappa^{2} C\left\|\mathcal{A} \boldsymbol{\xi}_{\boldsymbol{\sigma}}\right\|^{2}+\kappa^{2} C h^{2}\|\boldsymbol{\sigma}\|_{1}^{2}
$$

which completes the proof of (16). The proof of (17) follows similarly.

Now we estimate $G\left(\mathbf{u}, \mathbf{v}^{h}\right)-G_{h}\left(\mathbf{u}^{h}, \mathbf{v}^{h}\right)$. We assume that $\mathbf{u}$ is continuous in the whole domain for simplicity. Note that for $\mathbf{v}^{h} \in \mathbf{V}_{h}$, we have

$$
\begin{aligned}
G\left(\mathbf{u}, \mathbf{v}^{h}\right) & =\sum_{R} \int_{R}(\mathbf{b} \cdot \nabla \mathbf{u}) \cdot \mathbf{v}^{h} d s+\left(\alpha \mathbf{u}, \mathbf{v}^{h}\right) \\
& =\sum_{R} \int_{\partial R}(\mathbf{b} \cdot \mathbf{n}) \mathbf{u} \cdot \mathbf{v}^{h} d s+\left(\alpha \mathbf{u}, \mathbf{v}^{h}\right) .
\end{aligned}
$$

Thus, we find that

$$
\begin{aligned}
G\left(\mathbf{u}, \mathbf{v}^{h}\right)= & \sum_{R_{i, j}} \int_{\partial R_{i, j}}(\mathbf{b} \cdot \mathbf{n}) \mathbf{u} \cdot \mathbf{v}_{i, j}^{h} d s+\left(\alpha \mathbf{u}, \mathbf{v}^{h}\right) \\
= & \sum_{e_{i}^{x}} \int_{e_{i}^{x}}\left(\left(b_{1} n_{1}\right)^{+}+\left(b_{1} n_{1}\right)^{-}\right) \mathbf{u} \cdot\left(\mathbf{v}_{i-1, j}^{h}-\mathbf{v}_{i, j}^{h}\right) d s \\
& +\sum_{e_{j}^{y}} \int_{e_{j}^{y}}\left(\left(b_{2} n_{2}\right)^{+}+\left(b_{2} n_{2}\right)^{-}\right) \mathbf{u} \cdot\left(\mathbf{v}_{i, j-1}^{h}-\mathbf{v}_{i, j}^{h}\right) d s+\left(\alpha \mathbf{u}, \mathbf{v}^{h}\right)
\end{aligned}
$$

and

$$
\begin{aligned}
& G_{h}\left(\mathbf{u}^{h}, \mathbf{v}^{h}\right) \\
= & \sum_{R_{i, j}} \int_{\partial R_{i, j}}\left((\mathbf{b} \cdot \mathbf{n})^{+}\left(\mathbf{u}^{h}\right)^{\mathrm{int}}+(\mathbf{b} \cdot \mathbf{n})^{-}\left(\mathbf{u}^{h}\right)^{\mathrm{ext}}\right) \cdot \mathbf{v}_{i, j}^{h} d s+\left(\alpha \mathbf{u}^{h}, \mathbf{v}^{h}\right) \\
= & \sum_{e_{i}^{x}} \int_{e_{i}^{x}}\left(\left(b_{1} n_{1}\right)^{+} \mathbf{u}_{i-1, j}^{h}+\left(b_{1} n_{1}\right)^{-} \mathbf{u}_{i, j}^{h}\right) \cdot\left(\mathbf{v}_{i-1, j}^{h}-\mathbf{v}_{i, j}^{h}\right) d y \\
& +\sum_{e_{j}^{y}} \int_{e_{j}^{y}}\left(\left(b_{2} n_{2}\right)^{+} \mathbf{u}_{i, j-1}^{h}+\left(b_{2} n_{2}\right)^{-} \mathbf{u}_{i, j}^{h}\right) \cdot\left(\mathbf{v}_{i, j-1}^{h}-\mathbf{v}_{i, j}^{h}\right) d x+\left(\alpha \mathbf{u}^{h}, \mathbf{v}^{h}\right) .
\end{aligned}
$$

Writing $\mathbf{u}=\mathbf{P}_{h} \mathbf{u}+\left(\mathbf{u}-\mathbf{P}_{h} \mathbf{u}\right)$ and using

$$
\mathbf{P}_{h} \mathbf{u}_{i-1, j}+\left(\mathbf{u}-\mathbf{P}_{h} \mathbf{u}_{i-1, j}\right)=\mathbf{P}_{h} \mathbf{u}_{i, j}+\left(\mathbf{u}-\mathbf{P}_{h} \mathbf{u}_{i, j}\right),
$$


we arrive at

(20)

$$
\begin{aligned}
& G\left(\mathbf{u}, \mathbf{v}^{h}\right)-G_{h}\left(\mathbf{u}^{h}, \mathbf{v}^{h}\right)-G_{h}\left(\boldsymbol{\xi}_{\mathbf{u}}, \mathbf{v}^{h}\right)=\left(\alpha\left(\mathbf{u}-\mathbf{P}_{h} \mathbf{u}\right), \mathbf{v}^{h}\right) \\
& +\sum_{e_{i}^{x}} \int_{e_{i}^{x}}\left(\left(b_{1} n_{1}\right)^{+}\left(\mathbf{u}-\mathbf{P}_{h} \mathbf{u}_{i-1, j}\right)+\left(b_{1} n_{1}\right)^{-}\left(\mathbf{u}-\mathbf{P}_{h} \mathbf{u}_{i, j}\right)\right) \cdot\left(\mathbf{v}_{i-1, j}^{h}-\mathbf{v}_{i, j}^{h}\right) d y \\
& +\sum_{e_{j}^{y}} \int_{e_{j}^{y}}\left(\left(b_{2} n_{2}\right)^{+}\left(\mathbf{u}-\mathbf{P}_{h} \mathbf{u}_{i, j-1}\right)+\left(b_{2} n_{2}\right)^{-}\left(\mathbf{u}-\mathbf{P}_{h} \mathbf{u}_{i, j}\right)\right) \cdot\left(\mathbf{v}_{i, j-1}^{h}-\mathbf{v}_{i, j}^{h}\right) d x .
\end{aligned}
$$

Lemma 4.4. Under the assumption of Lemma 4.3 we have that

$$
\begin{aligned}
& G\left(\mathbf{u}, \boldsymbol{\xi}_{\mathbf{u}}\right)-G_{h}\left(\mathbf{u}^{h}, \boldsymbol{\xi}_{\mathbf{u}}\right)-G_{h}\left(\boldsymbol{\xi}_{\mathbf{u}}, \boldsymbol{\xi}_{\mathbf{u}}\right) \\
\leq & \kappa C h\|\mathbf{u}\|_{1}\left\|\mathcal{A} \boldsymbol{\xi}_{\boldsymbol{\sigma}}\right\|+\kappa C h^{2}\|\boldsymbol{\sigma}\|_{1}\|\mathbf{u}\|_{1}+\alpha C h\|\mathbf{u}\|_{1}\left\|\boldsymbol{\xi}_{\mathbf{u}}\right\| .
\end{aligned}
$$

Proof. Note that the unit outward normal vector is $\mathbf{n}= \pm(1,0)$ on $e_{i}^{x}$ and $\mathbf{n}= \pm(0,1)$ on $e_{j}^{y}$. By definition of projection, for $k=1,2$ the $L^{2}$-projection $P_{h} u_{k}$ is constant on $R_{i, j}$. So, it follows that

$$
\int_{e_{i}^{x}}\left(u_{k}-P_{h} u_{k}\right) d y=\frac{1}{h_{i}^{x}} \int_{R_{i, j}}\left(u_{k}\left(x_{i}, y\right)-u_{k}(x, y)\right) d x d y \leq \int_{R_{i, j}}\left|\frac{\partial u_{k}}{\partial x}\right| d x d y .
$$

By taking $\mathbf{v}^{h}=\boldsymbol{\xi}_{\mathbf{u}}$ in $(20)$, we have that

$$
\begin{aligned}
& \sum_{e_{i}^{x}} \int_{e_{i}^{x}}\left[\left(b_{1} n_{1}\right)^{+}\left(\mathbf{u}_{i-1, j}-\mathbf{P}_{h} \mathbf{u}_{i-1, j}\right)+\left(b_{1} n_{1}\right)^{-}\left(\mathbf{u}_{i, j}-\mathbf{P}_{h} \mathbf{u}_{i, j}\right)\right] \cdot\left(\mathbf{v}_{i-1, j}^{h}-\mathbf{v}_{i, j}^{h}\right) d y \\
& \leq C \sum_{e_{i}^{x}}\left(\int_{R_{i, j}}\left|\frac{\partial \mathbf{u}}{\partial x}\right| d x d y\left|\boldsymbol{\xi}_{\mathbf{u}_{i, j}}-\boldsymbol{\xi}_{\mathbf{u}_{i-1, j}}\right|\right) \\
& \leq C h\|\mathbf{u}\|_{1}\left[\sum_{e_{i}^{x}}\left|\boldsymbol{\xi}_{\mathbf{u}_{i, j}}-\boldsymbol{\xi}_{\mathbf{u}_{i-1, j}}\right|^{2}\right]^{1 / 2} \\
& \leq \kappa C h\|\mathbf{u}\|_{1}\left\|\mathcal{A} \boldsymbol{\xi}_{\boldsymbol{\sigma}}\right\|+\kappa C h^{2}\|\boldsymbol{\sigma}\|_{1}\|\mathbf{u}\|_{1} .
\end{aligned}
$$

Similarly by taking $\mathbf{v}^{h}=\boldsymbol{\xi}_{\mathbf{u}}$ in (20), we have

$$
\begin{aligned}
& \sum_{e_{j}^{y}} \int_{e_{j}^{y}}\left[\left(b_{2} n_{2}\right)^{+}\left(\mathbf{u}_{i, j-1}-\mathbf{P}_{h} \mathbf{u}_{i, j-1}\right)+\left(b_{2} n_{2}\right)^{-}\left(\mathbf{u}_{i, j}-\mathbf{P}_{h} \mathbf{u}_{i, j}\right)\right] \cdot\left(\mathbf{v}_{i, j-1}^{h}-\mathbf{v}_{i, j}^{h}\right) d x \\
& \leq C \sum_{e_{j}^{y}}\left(\int_{R_{i, j}}\left|\frac{\partial \mathbf{u}}{\partial y}\right| d x d y\left|\boldsymbol{\xi}_{\mathbf{u}_{i, j}}-\boldsymbol{\xi}_{\mathbf{u}_{i, j-1}}\right|\right) \\
& \leq \kappa C h\|\mathbf{u}\|_{1}\left\|\mathcal{A} \boldsymbol{\xi}_{\boldsymbol{\sigma}}\right\|+\kappa C h^{2}\|\boldsymbol{\sigma}\|_{1}\|\mathbf{u}\|_{1} .
\end{aligned}
$$


Since $\left\|\mathbf{u}-\mathbf{P}_{h} \mathbf{u}\right\| \leq C h\|\mathbf{u}\|_{1}$, adding the equation (21) and (22), we complete the proof from (20).

We are now ready to prove the first order convergence of the velocity and trace-free pseudostress variables.

Theorem 4.5. For $h$ sufficiently small, there exists a constant $C$ independent of $h$ such that

$$
\begin{aligned}
\left\|\mathcal{A}\left(\boldsymbol{\sigma}-\boldsymbol{\sigma}^{h}\right)\right\| & \leq C h\left(\|\boldsymbol{\sigma}\|_{1}+\|\mathbf{u}\|_{1}\right), \\
\left\|\mathbf{u}-\mathbf{u}^{h}\right\| & \leq C h\left(\|\boldsymbol{\sigma}\|_{1}+\|\mathbf{u}\|_{1}\right) .
\end{aligned}
$$

Proof. Note that $\boldsymbol{\sigma}-\boldsymbol{\sigma}^{h}=\boldsymbol{\eta}_{\boldsymbol{\sigma}}+\boldsymbol{\xi}_{\boldsymbol{\sigma}}$. Subtracting (8) from (6) and using (15), we have that

$$
\begin{cases}\left(\kappa \mathcal{A} \boldsymbol{\xi}_{\boldsymbol{\sigma}}, \boldsymbol{\tau}^{h}\right)+\left(\boldsymbol{\operatorname { d i v }} \boldsymbol{\tau}^{h}, \boldsymbol{\xi}_{\mathbf{u}}\right)+d\left(\boldsymbol{\tau}^{h}, \ell^{h}-\ell\right)=-\left(\kappa \mathcal{A} \boldsymbol{\eta}_{\boldsymbol{\sigma}}, \boldsymbol{\tau}^{h}\right), & \forall \boldsymbol{\tau}^{h} \in \mathbf{H}_{h}, \\ \left(\boldsymbol{\operatorname { d i v }} \boldsymbol{\xi}_{\boldsymbol{\sigma}}, \mathbf{v}^{h}\right)+G_{h}\left(\mathbf{u}^{h}, \mathbf{v}^{h}\right)-G\left(\mathbf{u}, \mathbf{v}^{h}\right)=0, & \forall \mathbf{v}^{h} \in \mathbf{V}_{h}, \\ d\left(\boldsymbol{\xi}_{\boldsymbol{\sigma}}, \mu^{h}\right)=0, & \forall \mu^{h} \in \mathbb{R} .\end{cases}
$$

Taking $\boldsymbol{\tau}^{h}=\boldsymbol{\xi}_{\boldsymbol{\sigma}}, \mathbf{v}^{h}=\boldsymbol{\xi}_{\mathbf{u}}$ and $\mu=\ell^{h}-\ell=0$, we have that

$$
\left\{\begin{array}{l}
\left(\kappa \mathcal{A} \boldsymbol{\xi}_{\boldsymbol{\sigma}}, \boldsymbol{\xi}_{\boldsymbol{\sigma}}\right)+\left(\operatorname{div} \boldsymbol{\xi}_{\boldsymbol{\sigma}}, \boldsymbol{\xi}_{\mathbf{u}}\right)=-\left(\kappa \mathcal{A} \boldsymbol{\eta}_{\boldsymbol{\sigma}}, \boldsymbol{\xi}_{\boldsymbol{\sigma}}\right), \\
\left(\operatorname{div} \boldsymbol{\xi}_{\boldsymbol{\sigma}}, \boldsymbol{\xi}_{\mathbf{u}}\right)+G_{h}\left(\mathbf{u}^{h}, \boldsymbol{\xi}_{\mathbf{u}}\right)-G\left(\mathbf{u}, \boldsymbol{\xi}_{\mathbf{u}}\right)=0
\end{array}\right.
$$

So, it follows that

$$
\begin{aligned}
\left(\kappa \mathcal{A} \boldsymbol{\xi}_{\boldsymbol{\sigma}}, \boldsymbol{\xi}_{\boldsymbol{\sigma}}\right)+G\left(\mathbf{u}, \boldsymbol{\xi}_{\mathbf{u}}\right)-G_{h}\left(\mathbf{u}^{h}, \boldsymbol{\xi}_{\mathbf{u}}\right) & =-\left(\kappa \mathcal{A} \boldsymbol{\eta}_{\boldsymbol{\sigma}}, \boldsymbol{\xi}_{\boldsymbol{\sigma}}\right) \\
& \leq \kappa C h\left\|\mathcal{A} \boldsymbol{\xi}_{\boldsymbol{\sigma}}\right\|\|\boldsymbol{\sigma}\|_{1}
\end{aligned}
$$

Note that from Lemma 3.2,

$$
\alpha\left\|\boldsymbol{\xi}_{\mathbf{u}}\right\|^{2} \leq G_{h}\left(\boldsymbol{\xi}_{\mathbf{u}}, \boldsymbol{\xi}_{\mathbf{u}}\right) .
$$

Applying (27) and Lemma 4.4, we have that

$$
\begin{aligned}
\kappa\left\|\mathcal{A} \boldsymbol{\xi}_{\boldsymbol{\sigma}}\right\|^{2}+\alpha\left\|\boldsymbol{\xi}_{\mathbf{u}}\right\|^{2} \leq & \left(\kappa \mathcal{A} \boldsymbol{\xi}_{\boldsymbol{\sigma}}, \boldsymbol{\xi}_{\boldsymbol{\sigma}}\right)+G_{h}\left(\boldsymbol{\xi}_{\mathbf{u}}, \boldsymbol{\xi}_{\mathbf{u}}\right) \\
\leq & {\left[\left(\kappa \mathcal{A} \boldsymbol{\xi}_{\boldsymbol{\sigma}}, \boldsymbol{\xi}_{\boldsymbol{\sigma}}\right)+G\left(\mathbf{u}, \boldsymbol{\xi}_{\mathbf{u}}\right)-G_{h}\left(\mathbf{u}^{h}, \boldsymbol{\xi}_{\mathbf{u}}\right)\right] } \\
& -\left[G\left(\mathbf{u}, \boldsymbol{\xi}_{\mathbf{u}}\right)-G_{h}\left(\mathbf{u}^{h}, \boldsymbol{\xi}_{\mathbf{u}}\right)-G_{h}\left(\boldsymbol{\xi}_{\mathbf{u}}, \boldsymbol{\xi}_{\mathbf{u}}\right)\right] \\
\leq & \kappa C h\left\|\mathcal{A} \boldsymbol{\xi}_{\boldsymbol{\sigma}}\right\|\|\boldsymbol{\sigma}\|_{1} \\
& +\kappa C h\|\mathbf{u}\|_{1}\left\|\mathcal{A} \boldsymbol{\xi}_{\boldsymbol{\sigma}}\right\|+\kappa C h^{2}\|\boldsymbol{\sigma}\|_{1}\|\mathbf{u}\|_{1}+\alpha C h\|\mathbf{u}\|_{1}\left\|\boldsymbol{\xi}_{\mathbf{u}}\right\| \\
\leq & \frac{1}{2} \kappa\left\|\mathcal{A} \boldsymbol{\xi}_{\boldsymbol{\sigma}}\right\|^{2}+\frac{1}{2} \alpha\left\|\boldsymbol{\xi}_{\mathbf{u}}\right\|^{2} \\
& +C h^{2}\left(\kappa\|\boldsymbol{\sigma}\|_{1}^{2}+(\kappa+\alpha)\|\mathbf{u}\|_{1}^{2}+\kappa\|\boldsymbol{\sigma}\|_{1}\|\mathbf{u}\|_{1}\right) .
\end{aligned}
$$

Thus,

$$
\kappa\left\|\mathcal{A} \boldsymbol{\xi}_{\boldsymbol{\sigma}}\right\|^{2}+\alpha\left\|\boldsymbol{\xi}_{\mathbf{u}}\right\|^{2} \leq C h^{2}\left(\kappa\|\boldsymbol{\sigma}\|_{1}^{2}+(\kappa+\alpha)\|\mathbf{u}\|_{1}^{2}+\kappa\|\boldsymbol{\sigma}\|_{1}\|\mathbf{u}\|_{1}\right) .
$$


Therefore, using $\left\|\mathcal{A}\left(\boldsymbol{\sigma}-\boldsymbol{\Pi}_{h} \boldsymbol{\sigma}\right)\right\| \leq C h\|\boldsymbol{\sigma}\|_{1}$ and $\left\|\mathbf{u}-\mathbf{P}_{h} \mathbf{u}\right\| \leq C h\|\mathbf{u}\|_{1}$ and the triangle inequality, we have

$$
\begin{aligned}
\left\|\mathcal{A}\left(\boldsymbol{\sigma}-\boldsymbol{\sigma}^{h}\right)\right\| & \leq C h\left(\|\boldsymbol{\sigma}\|_{1}+\|\mathbf{u}\|_{1}\right), \\
\left\|\mathbf{u}-\mathbf{u}^{h}\right\| & \leq C h\left(\|\boldsymbol{\sigma}\|_{1}+\|\mathbf{u}\|_{1}\right) .
\end{aligned}
$$

In the next theorem, we prove stability of $\boldsymbol{\sigma}_{\boldsymbol{h}}$ in the $\mathbf{H}(\mathbf{d i v} ; \Omega)$-norm. Note that in the scalar convection-diffusion problem, a weaker stability result was obtained [16]; see also Theorem 3.2 in [17].

Theorem 4.6. For $h$ sufficiently small, there exists a constant $C$ independent of $h$ such that

$$
\left\|\boldsymbol{\sigma}-\boldsymbol{\sigma}^{h}\right\|_{\mathbf{H}(\operatorname{div} ; \Omega)} \leq C\left(\|\boldsymbol{\sigma}\|_{1}+\|\mathbf{u}\|_{1}\right) .
$$

Proof. Consider error equation (25). Taking $\boldsymbol{\tau}^{h}=\boldsymbol{\xi}_{\boldsymbol{\sigma}}, \mathbf{v}^{h}=\operatorname{div} \boldsymbol{\xi}_{\boldsymbol{\sigma}}$ and $\mu=$ $\ell^{h}-\ell=0$, we get that

$$
\left\{\begin{array}{l}
\left(\kappa \mathcal{A} \boldsymbol{\xi}_{\boldsymbol{\sigma}}, \boldsymbol{\xi}_{\boldsymbol{\sigma}}\right)+\left(\operatorname{div} \boldsymbol{\xi}_{\boldsymbol{\sigma}}, \boldsymbol{\xi}_{\mathbf{u}}\right)=-\left(\kappa \mathcal{A} \boldsymbol{\eta}_{\boldsymbol{\sigma}}, \boldsymbol{\xi}_{\boldsymbol{\sigma}}\right), \\
\left(\operatorname{div} \boldsymbol{\xi}_{\boldsymbol{\sigma}}, \operatorname{div} \boldsymbol{\xi}_{\boldsymbol{\sigma}}\right)+G_{h}\left(\mathbf{u}^{h}, \operatorname{div} \boldsymbol{\xi}_{\boldsymbol{\sigma}}\right)-G\left(\mathbf{u}, \operatorname{div} \boldsymbol{\xi}_{\boldsymbol{\sigma}}\right)=0 .
\end{array}\right.
$$

Adding two equations in (29) leads to

$$
\begin{aligned}
& \left(\kappa \mathcal{A} \boldsymbol{\xi}_{\boldsymbol{\sigma}}, \boldsymbol{\xi}_{\boldsymbol{\sigma}}\right)+\left(\operatorname{div} \boldsymbol{\xi}_{\boldsymbol{\sigma}}, \operatorname{div} \boldsymbol{\xi}_{\boldsymbol{\sigma}}\right) \\
= & -\left(\kappa \mathcal{A} \boldsymbol{\eta}_{\boldsymbol{\sigma}}, \boldsymbol{\xi}_{\boldsymbol{\sigma}}\right)-\left(\operatorname{div} \boldsymbol{\xi}_{\boldsymbol{\sigma}}, \boldsymbol{\xi}_{\mathbf{u}}\right)+G\left(\mathbf{u}, \operatorname{div} \boldsymbol{\xi}_{\boldsymbol{\sigma}}\right)-G_{h}\left(\mathbf{u}^{h}, \operatorname{div} \boldsymbol{\xi}_{\boldsymbol{\sigma}}\right) .
\end{aligned}
$$

To estimate (30), using the relation

$$
\begin{aligned}
G\left(\mathbf{u}, \mathbf{v}^{h}\right) & =\sum_{R} \int_{\partial R}(\mathbf{b} \cdot \mathbf{n}) \mathbf{u} \cdot \mathbf{v}^{h} d s+\left(\alpha \mathbf{u}, \mathbf{v}^{h}\right) \\
& =\sum_{R} \int_{\partial R}\left((\mathbf{b} \cdot \mathbf{n})^{+} \mathbf{u}+(\mathbf{b} \cdot \mathbf{n})^{-} \mathbf{u}\right) \cdot \mathbf{v}^{h} d s+\left(\alpha \mathbf{u}, \mathbf{v}^{h}\right),
\end{aligned}
$$

we have

$$
\begin{aligned}
G\left(\mathbf{u}, \mathbf{v}^{h}\right)-G_{h}\left(\mathbf{u}^{h}, \mathbf{v}^{h}\right)= & \left(\alpha\left(\mathbf{u}-\mathbf{u}^{h}\right), \mathbf{v}^{h}\right) \\
& +\sum_{R} \int_{\partial R}(\mathbf{b} \cdot \mathbf{n})^{+}\left(\mathbf{u}-\left(\mathbf{u}^{h}\right)^{\mathrm{int}}\right) \cdot \mathbf{v}^{h} d s \\
& +\sum_{R} \int_{\partial R}(\mathbf{b} \cdot \mathbf{n})^{-}\left(\mathbf{u}-\left(\mathbf{u}^{h}\right)^{\mathrm{ext}}\right) \cdot \mathbf{v}^{h} d s \\
= & \left(\alpha\left(\mathbf{u}-\mathbf{u}^{h}\right), \mathbf{v}^{h}\right)+\mathrm{I}+\mathrm{II} .
\end{aligned}
$$

Note that $\mathbf{v}^{h}$ is a constant vector with support $R$.

$$
\begin{aligned}
\mathrm{I} & =\sum_{R} \int_{\partial R}(\mathbf{b} \cdot \mathbf{n})^{+}\left(\mathbf{u}-\left(\mathbf{u}^{h}\right)^{\mathrm{int}}\right) \cdot \mathbf{v}^{h} d s \\
& \leq C \sum_{R}\left|\mathbf{v}^{h}\right| \int_{\partial R}\left|\mathbf{u}-\mathbf{u}^{h}\right| d s
\end{aligned}
$$




$$
\begin{aligned}
& \leq C \sum_{R}\left|\mathbf{v}^{h}\right| h^{1 / 2}\left(\int_{\partial R}\left|\mathbf{u}-\mathbf{u}^{h}\right|^{2} d s\right)^{1 / 2} \\
& \leq C h^{-1 / 2} \sum_{R}\left(h\left|\mathbf{v}^{h}\right|\right)\left(\left\|\mathbf{u}-\mathbf{u}^{h}\right\|_{0, \partial R}\right) \\
& \leq C h^{-1 / 2}\left(\sum_{R} h^{2}\left|\mathbf{v}^{h}\right|^{2}\right)^{1 / 2}\left(\sum_{R}\left\|\mathbf{u}-\mathbf{u}^{h}\right\|_{0, \partial R}^{2}\right)^{1 / 2} .
\end{aligned}
$$

By the trace theorem(c.f., [3]) and Theorem 4.5,

$$
\begin{aligned}
\mathrm{I} & \leq C h^{-1 / 2}\left\|\mathbf{v}^{h}\right\|_{0}\left(\sum_{R}\left\|\mathbf{u}-\mathbf{u}^{h}\right\|_{0, R}\left\|\mathbf{u}-\mathbf{u}^{h}\right\|_{1, R}\right)^{1 / 2} \\
& \leq C h^{-1 / 2}\left(\left\|\mathbf{u}-\mathbf{u}^{h}\right\|_{0}\|\mathbf{u}\|_{1}\right)^{1 / 2}\left\|\mathbf{v}^{h}\right\|_{0} \\
& \leq C\left(\|\boldsymbol{\sigma}\|_{1}+\|\mathbf{u}\|_{1}\right)\left\|\mathbf{v}^{h}\right\|_{0} .
\end{aligned}
$$

Similarly, we get

$$
\mathrm{II} \leq C\left(\|\boldsymbol{\sigma}\|_{1}+\|\mathbf{u}\|_{1}\right)\left\|\mathbf{v}^{h}\right\|_{0} .
$$

Thus, taking $\mathbf{v}^{h}=\operatorname{div} \boldsymbol{\xi}_{\sigma}$,

$$
\left|G\left(\mathbf{u}, \operatorname{div} \boldsymbol{\xi}_{\boldsymbol{\sigma}}\right)-G_{h}\left(\mathbf{u}^{h}, \operatorname{div} \boldsymbol{\xi}_{\boldsymbol{\sigma}}\right)\right| \leq C\left(\|\boldsymbol{\sigma}\|_{1}+\|\mathbf{u}\|_{1}\right)\left\|\operatorname{div} \boldsymbol{\xi}_{\boldsymbol{\sigma}}\right\|_{0} .
$$

Therefore, we get the following estimate from (7) and (30)

$$
\begin{aligned}
\left\|\boldsymbol{\xi}_{\sigma}\right\|_{\mathbf{H}(\operatorname{div} ; \Omega)}^{2} & =\left\|\boldsymbol{\xi}_{\sigma}\right\|^{2}+\left\|\operatorname{div} \boldsymbol{\xi}_{\sigma}\right\|^{2} \\
& \leq C\left(\left\|\mathcal{A} \boldsymbol{\xi}_{\sigma}\right\|^{2}+\left\|\operatorname{div} \boldsymbol{\xi}_{\sigma}\right\|^{2}\right) \\
& \leq C\left(\left\|\mathcal{A} \eta_{\sigma}\right\|\left\|\boldsymbol{\xi}_{\sigma}\right\|+\left\|\boldsymbol{\xi}_{\mathbf{u}}\right\|\left\|\operatorname{div} \boldsymbol{\xi}_{\sigma}\right\|+\left(\|\boldsymbol{\sigma}\|_{1}+\|\mathbf{u}\|_{1}\right)\left\|\operatorname{div} \boldsymbol{\xi}_{\sigma}\right\|\right) \\
& \leq C\left(\left\|\mathcal{A} \eta_{\sigma}\right\|+\left\|\boldsymbol{\xi}_{\mathbf{u}}\right\|+\|\boldsymbol{\sigma}\|_{1}+\|\mathbf{u}\|_{1}\right)\left\|\boldsymbol{\xi}_{\boldsymbol{\sigma}}\right\|_{\mathbf{H}(\operatorname{div} ; \Omega)} .
\end{aligned}
$$

By the triangle inequality

$$
\left\|\boldsymbol{\sigma}-\boldsymbol{\sigma}_{h}\right\|_{\mathbf{H}(\mathrm{div} ; \Omega)} \leq\left\|\boldsymbol{\eta}_{\sigma}\right\|_{\mathbf{H}(\mathrm{div} ; \Omega)}+\left\|\boldsymbol{\xi}_{\sigma}\right\|_{\mathbf{H}(\mathbf{d i v} ; \Omega)} \leq C\left(\|\boldsymbol{\sigma}\|_{1}+\|\mathbf{u}\|_{1}\right) .
$$

\section{Numerical results}

In this section, we perform various numerical experiments to test the upstream scheme based on the pseudostress-velocity formulation. All experiments were run in Matlab (see [1]).

\subsection{Example 1}

We solve Oseen equations ( 1 ) in the unit square $\Omega=(0,1)^{2}$ with $\alpha=2$ and $\mathbf{b}=(2,3)^{T}$. The function $\mathbf{f}$ is determined by the following exact solution,

$$
\mathbf{u}=\left(\begin{array}{c}
\pi \sin (\pi x)^{2} \sin (2 \pi y) \\
-\pi \sin (2 \pi x) \sin (\pi y)^{2}
\end{array}\right), \quad p(x, y)=\cos (\pi x) \cos (\pi y)
$$


By the definition of the pseudostress in (2), we have

$$
\begin{aligned}
\boldsymbol{\sigma} & =\nu \nabla \mathbf{u}-p \mathbf{I} \\
& =\left(\begin{array}{cc}
\nu \pi^{2} \sin (2 \pi x) \sin (2 \pi y)-p(x, y) & 2 \nu \pi^{2} \sin ^{2}(\pi x) \cos (2 \pi y) \\
-2 \nu \pi^{2} \cos (2 \pi x) \sin ^{2}(\pi y) & -\nu \pi^{2} \sin (2 \pi x) \sin (2 \pi y)-p(x, y)
\end{array}\right) .
\end{aligned}
$$

Partition the domain $\Omega=(0,1)^{2}$ by uniform rectangular elements $R_{i, j}=$ $(i h, j h)$ for $i, j=0,1, \ldots, n$ with $h=1 / n$. When $n=32$, Figure 2 shows the exact vector field of velocity and contours of pressure, respectively. If we write

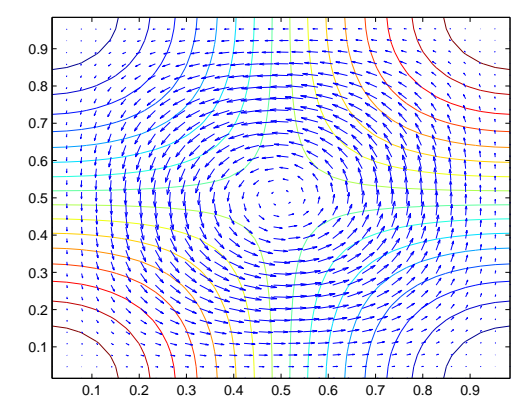

FiguRE 2. Exact vector field of velocity and contours of pressure

the pseudostress $\boldsymbol{\sigma}$ and velocity $\mathbf{u}$ as

$$
\boldsymbol{\sigma}=\sum_{j=1}^{M} \Sigma_{j} \boldsymbol{\Psi}_{j}, \quad \mathbf{u}=\sum_{j=1}^{N} U_{j} \phi_{j},
$$

where $M=4 n(n+1), N=2 n^{2}$. The discrete weak form (8) has the following matrix form:

$$
\left[\begin{array}{ccc}
B & C^{T} & E^{T} \\
C & -G & 0 \\
E & 0 & 0
\end{array}\right]\left[\begin{array}{c}
\Sigma \\
U \\
\ell
\end{array}\right]=\left[\begin{array}{c}
0 \\
-F \\
0
\end{array}\right]
$$

Note that $\operatorname{rank}(B)=M-1$ and $G$ is positive definite by Lemma 3.2. If we choose $\nu$ smaller, the Oseen equations become more convection-dominated. The table 1 displays the $L^{2}$-norm errors of $\mathcal{A} \boldsymbol{\sigma}$ and velocity $\mathbf{u}$ and their convergence orders (C.O.) compared with $L^{2}$-norm and $\mathbf{H}(\mathbf{d i v} ; \Omega)$-norm errors for $\boldsymbol{\sigma}$. From the table we confirm our theory presented in this paper.

\subsection{Lid-driven cavity flow}

The next problem is that of lid-driven flow in a square cavity. This is a classic test problem used in fluid dynamics, known as dirven-cavity flow. Our aim here is to check the performance of the scheme with $\mathbf{b}=\mathbf{0}$. We 
TABlE 1. Errors and Convergence Orders

\begin{tabular}{|c|c|c|c|c|c|c|c|c|}
\hline $\mathrm{N}$ & $\left|\mathcal{A}\left(\boldsymbol{\sigma}-\boldsymbol{\sigma}^{h}\right)\right| \mid$ & C.O. & $\left|\mathbf{u}-\mathbf{u}^{h}\right|$ & C.O. & $\mid \boldsymbol{\sigma}-\boldsymbol{\sigma}^{h}$ & C.O. & $\mid \boldsymbol{\sigma}-\boldsymbol{\sigma}^{h} \|_{\mathbf{H}(\operatorname{div} ; \Omega)}$ & C.O. \\
\hline$\nu=1$ & & & & & & & & \\
\hline 4 & 5.7847 & * & 1.0800 & * & 6.1823 & * & 62.0662 & * \\
\hline 8 & 2.9490 & 0.97 & 0.5726 & 0.92 & 3.2408 & 0.93 & 34.1748 & 0.86 \\
\hline 16 & 1.4605 & 1.01 & 0.2892 & 0.99 & 1.6213 & 1.00 & 17.9315 & 0.93 \\
\hline 32 & 0.7203 & 1.02 & 0.1445 & 1.00 & 0.8061 & 1.01 & 9.3534 & 0.94 \\
\hline 64 & 0.3566 & 1.01 & 0.0722 & 1.00 & 0.4016 & 1.01 & 4.9647 & 0.91 \\
\hline$\nu=0.1$ & & & & & & & & \\
\hline 4 & 1.0358 & * & 1.3760 & * & 1.4094 & * & 10.8401 & * \\
\hline 8 & 0.6717 & 0.62 & 0.8410 & 0.71 & 1.2398 & 0.19 & 8.3898 & 0.37 \\
\hline 16 & 0.3947 & 0.77 & 0.4760 & 0.82 & 0.8637 & 0.52 & 6.2219 & 0.43 \\
\hline 32 & 0.2186 & 0.85 & 0.2573 & 0.89 & 0.5193 & 0.73 & 4.3634 & 0.51 \\
\hline 64 & 0.1165 & 0.91 & 0.1349 & 0.93 & 0.2873 & 0.85 & 2.9226 & 0.58 \\
\hline$\nu=0.01$ & & & & & & & & \\
\hline 4 & 0.1373 & * & 1.5516 & * & 0.7004 & * & 2.7386 & $*$ \\
\hline 8 & 0.1085 & 0.34 & 0.9647 & 0.69 & 0.7285 & -0.06 & 3.2025 & -0.23 \\
\hline 16 & 0.0747 & 0.54 & 0.5536 & 0.80 & 0.6578 & 0.15 & 3.4616 & -0.11 \\
\hline 32 & 0.0454 & 0.72 & 0.3014 & 0.88 & 0.4600 & 0.52 & 3.0307 & 0.19 \\
\hline 64 & 0.0264 & 0.78 & 0.1587 & 0.93 & 0.2718 & 0.76 & 2.6118 & 0.21 \\
\hline$\nu=0.001$ & & & & & & & & \\
\hline 4 & 0.0145 & * & 1.5820 & $*$ & 0.7041 & * & 2.3247 & $*$ \\
\hline 8 & 0.0136 & 0.09 & 1.0003 & 0.66 & 0.7023 & 0.00 & 2.3364 & -0.01 \\
\hline 16 & 0.0141 & -0.05 & 0.5879 & 0.77 & 0.6734 & 0.06 & 2.3478 & -0.01 \\
\hline 32 & 0.0112 & 0.34 & 0.3195 & 0.88 & 0.5388 & 0.32 & 2.0837 & 0.17 \\
\hline 64 & 0.0071 & 0.66 & 0.1655 & 0.95 & 0.3180 & 0.76 & 1.4674 & 0.51 \\
\hline
\end{tabular}

impose no-slip boundary conditions, that is, $u_{1}(x, 1)=1$ for $-1<x<1$ and $u_{1}(-1,1)=u_{1}(1,1)=0$. We solve the following Stokes equations with uniform mesh.

$$
\left\{\begin{array}{l}
-\triangle \mathbf{u}+\nabla p=\mathbf{0} \text { in }(-1,1) \times(-1,1) \\
\operatorname{div} \mathbf{u}=0 \text { in }(-1,1) \times(-1,1) .
\end{array}\right.
$$

We plot exponentially spaced streamlines to illustrate the Moffatt eddies in the bottom corners. These streamlines are computed from the pseudostress solution by solving the following Poisson equation numerically subject to a zero Dirichlet boundary condition.

$$
-\nabla^{2} \phi=\frac{\partial u_{2}}{\partial x}-\frac{\partial u_{1}}{\partial y}:=\omega
$$

where $\phi$ is a scalar stream function and $\omega$ is the vorticity (see [13, 21]). Because our method is based on the pseudostress-velocity formulation we calculate the pseudostress directly. Since

$$
\kappa \mathcal{A} \boldsymbol{\sigma}=\nabla \mathbf{u}=\left(\begin{array}{cc}
\frac{\partial u_{1}}{\partial x} & \frac{\partial u_{1}}{\partial y} \\
\frac{\partial u_{2}}{\partial x} & \frac{\partial u_{2}}{\partial y}
\end{array}\right)
$$




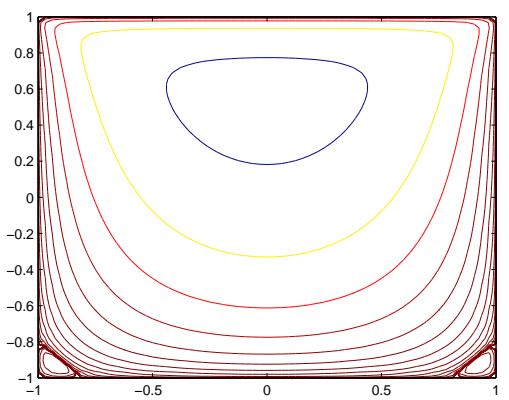

FiguRE 3. Contours of the stream function with exponentially distributed

we can use more accurate approximation of $\omega$ from the computed pseudostress so that we shall solve equation (31) more efficiently.

\subsection{Oseen flow over a step}

The final test problem is an Oseen flow over a backward facing step.

$$
\left\{\begin{array}{l}
-\frac{1}{R e} \Delta \mathbf{u}+\mathbf{b} \cdot \nabla \mathbf{u}+\nabla p=\mathbf{0} \text { in } \Omega, \\
\operatorname{div} \mathbf{u}=0 \text { in } \Omega .
\end{array}\right.
$$

The domain $\Omega$ is L-shape as Figure 4. The Reynolds number Re is 100 and we impose a constant left-to-right wind $\mathbf{b}=(1,0)$ for $y \geq 0$ and $\mathbf{b}=(0,0)$ for $y<0$. Inflow velocity is $u_{1}(-1, y)=y(1-y)$ for $0<y<1$. Outflow boundary condition is

$$
\left\{\begin{array}{l}
-p+\frac{1}{\operatorname{Re}} \frac{\partial u_{1}}{\partial x}=0, \\
\frac{\partial u_{2}}{\partial x}=0 .
\end{array}\right.
$$

This condition is equivalent to $\boldsymbol{\sigma} \mathbf{n}=\mathbf{0}$. The other boundary velocities are all zero. All above conditions are depicted in Figure 4.

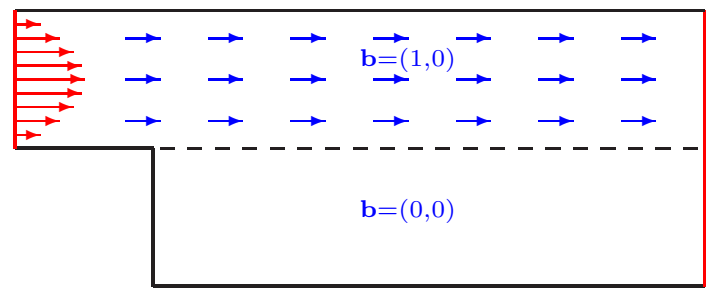

Figure 4. Domain and boundary conditions 
We compute the approximation solution of equations (32) with pseudostressvelocity formulation involving our upstream method. From the velocity computed already, the streamlines are plotted by Matlab automatically.

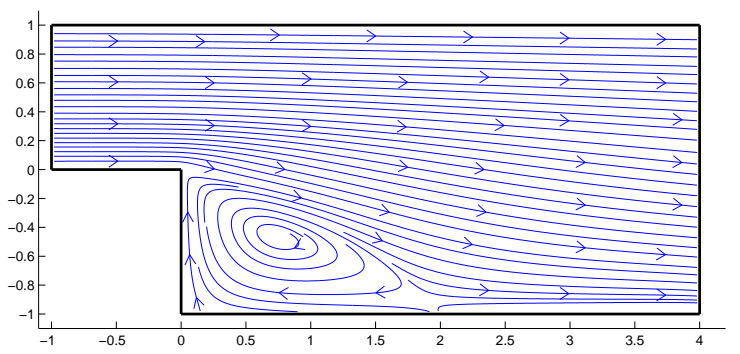

Figure 5. The streamlines when Re is 100

\section{References}

[1] C. Bahriawati and C. Carstensen, Three Matlab implementations of the lowest-order Raviart-Thomas MFEM with a posteriori error control, Comput. Methods Appl. Math. 5 (2005), no. 4, 333-361.

[2] M. Braack and E. Burman, Local projection stabilization for the Oseen problem and its interpretation as a variational multiscale method, SIAM J. Numer. Anal. 43 (2006), no. 6, 2544-2566.

[3] S. Brenner and L. R. Scott, The Mathematical Theory of Finite Element Methods, Springer-Verlag, New York, 1994.

[4] F. Brezzi and M. Fortin, Mixed and Hybrid Finite Element Methods, Springer-Verlag, New York, 1991.

[5] A. N. Brooks and T. J. R. Hughes, Streamline upwind/Petrov-Galerkin formulations for convection dominated flows with particular emphasis on the incompressible NavierStokes equations, Comput. Methods Appl. Mech. Engrg. 32 (1982), no. 1-3, 199-259.

[6] E. Burman, M. A. Fernandez, and P. Hansbo, Continuous interior penalty finite element method for Oseen's equations, SIAM J. Numer. Anal. 44 (2006), no. 3, 1248-1274.

[7] Z. Cai and G. Starke, First-order system least squares for the stress-displacement formulation: linear elasticity, SIAM J. Numer. Anal. 41 (2003), no. 2, 715-730.

[8] Z. Cai, C. Tong, P. S. Vassilevski, and C. Wang, Mixed finite element methods for incompressible flow: Stationary Stokes equations, Numer. Methods Partial Differential Equations 26 (2010), no. 4, 957-978.

[9] Z. Cai and Y. Wang, Pseudostress-velocity formulation for incompressible Navier-Stokes equations, Internat. J. Numer. Methods Fluids 63 (2010), no. 3, 341-356.

[10] C. Carstensen, D. Kim, and E.-J. Park, A priori and a posteriori pseudostress-velocity mixed finite element error analysis for the Stokes problem, SIAM J. Numer. Anal. 49 (2011), no. 6, 2501-2523.

[11] S. H. Chou, Mixed upwinding covolume methods on rectangular grids for convectiondiffusion problems, SIAM J. Sci. Comput. 21 (1999), no. 1, 145-165.

[12] C. Dawson, Analysis of an upwind-mixed finite element method for nonlinear contaminant transport equations, SIAM J. Numer. Anal. 35 (1998), no. 5, 1709-1724. 
[13] H. Elman, D. Silvester, and A. Wathen, Finite Elements and Fast Iterative Solvers: With applications in incompressible fluid dynamics, Oxford University Press, New York, 2005.

[14] G. Gatica, A. Marquez, and M. A. Sanchez, Analysis of a velocity-pressure-pseudostress formulation for the stationary Stokes equations, Comput. Methods Appl. Mech. Engrg. 199 (2010), no. 17-20, 1064-1079.

[15] V. Girault and P. A. Raviart, Finite Element Methods for Navier-Stokes Equations, Springer-Verlag, New York, 1986.

[16] J. Jaffre, Eléments finis mixtes et décentrage pour les équations de diffusion-convection, Calcolo 23 (1984), 171-197.

[17] D. Kim and E.-J. Park, A posteriori error estimators for the upstream weighting mixed methods for convection diffusion problems, Comput. Methods Appl. Mech. Engrg. 197 (2008), no. 6-8, 806-820.

[18] P. Lesaint and P. A. Raviart, On a finite element method for solving the neutron trasport equation, Mathematical Aspect of Finite Elements in Partial Differential Equations, Ed. Carl de Boor, Academic Press, 1974, 89-123.

[19] Z. Li, Convergence analysis of an upwind mixed element method for advection diffusion problems, Appl. Math. Comput. 212 (2009), no. 2, 318-326.

[20] F. A. Milner and E.-J. Park, A mixed finite element method for a strongly nonlinear second-order elliptic problem, Math. Comp. 64 (1995), no. 211, 973-988.

[21] S. Norburn and D. Silvester, Stabilised vs. stable mixed methods for incompressible flow, Comput. Methods Appl. Mech. Engrg. 166 (1998), no. 1-2, 131-141.

[22] E.-J. Park, Mixed finite element methods for nonlinear second order elliptic problems, SIAM J. Numer. Anal. 32 (1995), no. 3, 865-885.

[23] P. A. Raviart and J. Thomas, A mixed finite element method for 2nd order elliptic problems, Mathematical aspects of finite element methods (Proc. Conf., Consiglio Naz. delle Ricerche (C.N.R.), Rome, 1975), pp. 292315. Lecture Notes in Math., Vol. 606, Springer, Berlin, 1977.

EUN-JAE PARK

Department of Mathematics and

Department of Computational Science and Engineering

YoNSEI UNIVERSITY

SEOUl 120-749, KoreA

E-mail address: ejpark@yonsei.ac.kr

BOYOON SEO

Department of Mathematics

Yonsei UNIVERSITY

SEOUl 120-749, KoreA

E-mail address: mathied@yonsei.ac.kr 